\title{
TOPICAL DELIVERY OF QUERCETIN LOADED TRANSFERSOMES FOR WOUND TREATMENT: IN VITRO AND IN VIVO EVALUATION
}

\author{
MARWA ABDALLAH ${ }^{1 *}$, DEMIANA I. NESSEEM ${ }^{1}$, OMAIMA N. ELGAZAYERLY², ALY A. ABDELBARY ${ }^{2,3}$ \\ ${ }^{1}$ National Organization of Drug Control and Research, Pharmaceutics Department, Egypt, ${ }^{2}$ Department of Pharmaceutics and Industrial \\ Pharmacy, Faculty of Pharmacy, Cairo University, Cairo, Egypt, ${ }^{3}$ School of Life and Medical Sciences, University of Hertfordshire hosted by \\ Global Academic Foundation, Cairo, Egypt \\ Email: dr_maramiro@msn.com
}

Received: 14 Mar 2021, Revised and Accepted: 13 Jul 2021

\section{ABSTRACT}

Objective: To design topical Quercetin (Qc)-loaded transfersomes (TFs) for wound treatment.

Methods: Qc-loaded TFs were prepared by thin-film hydration technique using $2^{2} 4^{1}$ full factorial design and the optimum formula was selected. In vivo skin, deposition and cutaneous wound induction studies were performed for four groups of male wistar rats. At the end of the experiment, biochemical parameters were measured in the healed tissues (total proteins (TP), total antioxidant capacity (TAC), glutathione reductase (GSH), nitric oxide (NO), and malonaldehyde (MDA). Two in vivo histopathological experiments using male wistar rats were performed; the first study was done for the healed tissues of the above experiment and the second was to confirm the safety of formulations.

Results: Qc optimum TFs (F6) showed EE\% of 91.1\%, PS of $695.35 \mathrm{~nm}$, PDI of 0.592, and ZP of-11.1 mV, and spherical shape. In vivo skin deposition study showed that drug percentage retained in the skin from Qc optimum TFs was significantly higher than that from Qc suspension and Qc liposomes $(\mathrm{p}<0.05)$. There was no significant difference in the values of TP, TAC and MDA between the treated groups ( $p>0.05)$. GSH in TFs treated groups was significantly higher than the other groups $(\mathrm{p}<0.05)$ while NO in TFs treated groups was significantly lower than the other treated groups $(\mathrm{p}<0.05)$. Histopathological experiments showed that wounds treated by TFs healed better than those treated by both liposomes and Qc suspension.

Conclusion: Qc-loaded TFs can be used as successful drug-delivery system for wound healing.

Keywords: Quercetin, Transfersomes, Wound treatment, Topical formulation

(C) 2021 The Authors. Published by Innovare Academic Sciences Pvt Ltd. This is an open access article under the CC BY license (https://creativecommons.org/licenses/by/4.0/) DOI: https://dx.doi.org/10.22159/ijap.2021v13i5.41435. Journal homepage: https://innovareacademics.in/journals/index.php/ijap

\section{INTRODUCTION}

Herbal drugs have been used in many diseases [1]. Plant pigments which are found in herbal drugs as flavonoids which are also found in fruits and vegetables, have many physiological activities [2]. Flavonoids are important drug candidates due to their free radical scavenging and anti-inflammatory properties $[3,4]$. There is a structure-activity relationship between several flavonoids (luteolin, kaempferol, and chrysin) and their antioxidant activity and the number of $\mathrm{OH}$ groups in their structure so they are used in inflammatory disorders such as psoriasis and arthritis [5].

Quercetin (Qc) has high antioxidant activity which reduces the expression of matrix metalloproteinase that causes wrinkling and decreasing of the elasticity of healthy and photoaged skin [6]. Qc has antioxidant and antiradical effects due to three functional groups in its structure [7-9]. Nanotechnology is urgently needed for herbal drugs due to the poor solubility of those drugs [10]. The topical application of Qc in various formulation approaches includes permeation enhancers [11] such as lecithin and chitosan nanoparticles [12] which increase the penetration of Qc through the skin to make its topical and transdermal delivery easier, targeting the drug towards the desired tissue and concentrating the drug in it while reducing its concentration in the non-target tissues. Hence, drug action increases and side effects decrease [13]. Topical dosage forms are superior drug delivery systems because they avoid firstpass hepatic metabolism, gastric degradation and improve penetration of the drug into skin layers $[14,15]$. Surface-active agent vesicles are an alternative drug delivery system to conventional liposomes. Transfersomes (TFs) are elastic nano-vesicles composed of lecithin and surface-active agent and they differ from the conventional liposomes by their ability to squeeze themselves into the intercellular region of the stratum corneum $[16,17]$.

This study aimed to develop topical Qc-loaded TFs for wound treatment. Optimization of production parameters to produce stable, highly concentrated Qc vesicles with a small particle size was done using full factorial statistical design. In addition, in vivo skin deposition of Qc from optimum TFs was compared with Qc liposomes and Qc suspension using male Wistar rats. In addition, wound induction was done to male Wistar rats which were then treated with Qc optimum TFs, Qc liposomes, and Qc suspension by measuring the biological parameters in the treated skin tissues of rats. Furthermore, two in vivo histopathological experiments were performed; the first to compare the wound treatment effect of Qc optimum TFs, Qc liposomes, and Qc suspension. The second in vivo histopathological experiment was performed to assess the irritation potential of Qc optimum TFs on rat skin.

\section{MATERIALS AND METHODS}

\section{Materials}

Quercetin (Qc) dihydrate 97\%, Cholesterol 95\%, and Polysorbate 60 (Tween 60) were purchased from ALFA Aesar (A Johnson Matthey Company), Germany. Ethanol absolute was purchased from Sigma Aldrich, Germany. Chloroform was purchased from Sigma Aldrich, United Kingdom. Sodium Chloride, Potassium chloride, Disodium hydrogen orthophosphate, Potassium dihydrogen orthophosphate, and Tween 80 were purchased from ADWIC, ELNASR pharmaceutical chemicals company, Egypt. Span 60 and Span 40 were purchased from LOBA Chemie, India. Lecithin granular from egg yolk was purchased from Acros organics, USA. Sorbitan monooleate (Span80) was purchased from MP Biomedicals, France. Glutathione reduced (GSH), Malonaldehyde (MDA), Nitric oxide (NO), Total proteins (TP) and Total antioxidant capacity (TAC) kits colorimetric method were purchased from Biodiagnostic and research reagents, Egypt.

\section{Experimental design}

The design used was complete factorial $2^{2} 4^{1}$, which studied the influence of different variables on Qc-loaded TFs [18]. One factor was assessed with 4 levels ( $\mathrm{X}_{1}$ : Type of the surface-active agent), while the others with two levels $\left(\mathrm{X}_{2}\right.$ : Lecithin to surface-active agent

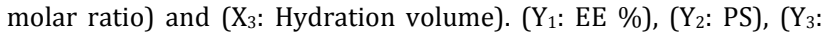


$\mathrm{PDI})$ and $\left(\mathrm{Y}_{4}: \mathrm{ZP}\right)$ were selected as the dependent variables. Table 1 shows the composition of the prepared TFs suspensions. Design
Expert ${ }^{\circledR}$ software was used to calculate desirability for the selection of the optimum formula.

Table 1: Full factorial design $\left(2^{2} 4^{1}\right)$ used for choosing optimum formulation of the TFs formulations

\begin{tabular}{|c|c|c|c|}
\hline Factors of independent variables & Levels & & \\
\hline $\mathrm{X}_{1}$ & Tween 60 & Tween 80 & Span60 \\
\hline $\mathrm{X}_{2}$ & Span 80 & & \\
\hline $\mathrm{X}_{3}$ & $5: 1$ & $10: 1$ & \\
\hline & $10 \mathrm{ml}$ & $20 \mathrm{ml}$ & \\
\hline Responses of dependent variables & Desirability & & \\
\hline $\mathrm{Y}_{1}: \mathrm{EE} \%$ & Maximize & & \\
\hline $\mathrm{Y}_{2}: \mathrm{PS}(\mathrm{nm})$ & Minimize & & \\
\hline $\mathrm{Y}_{3}: \mathrm{PDI}$ & Minimize & & \\
\hline $\mathrm{Y}_{4}: \mathrm{ZP}(\mathrm{mV})$ & Maximize (as absolute value) & & \\
\hline
\end{tabular}

Abbreviations: EE\%, entrapment efficiency percent; PS, particle size; PDI, polydispersity index; and ZP, zeta potential.

\section{Preparation by thin-film hydration technique}

Preparation of TFs was performed by thin-film hydration technique [19] as follows: specified weights of Qc, lecithin from egg yolk, Span 60, Span 80, Tween 60, Tween 80 were dissolved in $20 \mathrm{ml}$ (chloroform and ethanol, $1: 1 \mathrm{v} / \mathrm{v}$ ) then were put in a rotaryevaporator (Rotavapor, Heidolph VV 2000, Burladingen, Germany). The solvent was evaporated at $60^{\circ} \mathrm{C}$, then the thin layer formed was rehydrated by saline phosphate buffer at $60{ }^{\circ} \mathrm{C}$ with agitation using glass beads followed by sonication. To compare the permeation of the prepared TFs and other vesicles; Qc liposomes (composed of Qc and lecithin from egg yolk) were prepared in the same method and amounts as above.

\section{Qc loaded TFs characterization}

\section{Measurement of PS, PDI, and ZP}

Measurement of PS (z-average), PDI, and ZP of the prepared TFs suspensions was performed by Photon Correlation Spectroscopy (PCS) using a Zetasizer Nano ZS-90 instrument (Malvern Instruments, Worcestershire, UK). TFs suspensions were diluted before the measurement. Measurements were performed in triplicate using a $90{ }^{\circ} \mathrm{C}$ scattering angle at $25{ }^{\circ} \mathrm{C}$. Conversion of electrophoretic mobility to zeta potential was calculated by the Smoluchowski equation [20]. The viscosity of the samples was considered to be that of water [21]. Results are the average value \pm SD.

\section{Determination of Qc EE\% in TFs}

The difference between the total amount of Qc added in the formulation and that remaining in the aqueous medium, after separating the TFs suspension by centrifugation at $8500 \mathrm{rpm}$ for 30 min using a centrifuge (Biofuge primo Heraeus instruments, Germany), was calculated to determine the EE\% of Qc TFs. TFs contents were determined spectrophotometrically at a wavelength of $372 \mathrm{~nm}$. EE\% was calculated using the equation:

$$
\mathrm{EE}(\%)=\left[\left(\frac{\text { Total amount of } \mathrm{Qc} \text { added }- \text { amount of free } \mathrm{Qc}}{\text { Total amount of } \mathrm{Qc} \text { added }}\right)\right] \times 100
$$

\section{Formulation optimization}

The optimum formula was obtained using the Design Expert ${ }^{\circledR}$ software by applying constraints on encapsulation efficiency percent of the TFs to reach the maximum value, ZP to obtain the maximum as an absolute value and on particle size and PDI to obtain the smallest value.

\section{TEM}

The particle shape of the Qc optimum TFs suspension was visualized using JEM-2100 Electron Microscope, Japan. A drop of TFs suspension was placed onto a film-coated copper grid, to make a thin film, before the specimen was dried on the grid. A drop of $1 \%$ phosphotungstic acid was used to stain the above film. The grid was then viewed at an accelerating voltage of $200 \mathrm{Kv}$ [22].

\section{DSC}

DSC (Shimadzu DSC-50, Japan) calibrated with purified medium $(99.9 \%)$ was used to perform thermal analysis of Qc, lecithin egg yolk, Tween 80, physical mixture of Qc with TFs components, and the Qc optimum TFs. Mounting the sample was done in standard aluminum pans and a temperature of range $10-35{ }^{\circ} \mathrm{C}$ was used to heat it at a scanning rate of $10{ }^{\circ} \mathrm{C} / \mathrm{min}$ inert nitrogen flow $(25$ $\mathrm{ml} / \mathrm{min}$ ).

\section{In vivo assessment of Qc loaded TFs}

The ethical committee of the Faculty of Pharmacy, Cairo University (PT 1428) approved the in vivo experiment. Male Wistar rats weighing $(150-200 \mathrm{~g})$ were obtained from the farm of the National Organization for Drug Control and Research, Giza, Egypt. Rats were maintained on a basal diet. Animals were treated according to the guidelines of Association of Official Analytical Chemists (AOAC) [23].

\section{In vivo skin deposition experiment}

Seventy-two male Wistar rats were used in the experiment. Four groups of 18 rats each were used. Group (I) acted as a control group, group (II), (III), and (IV) received topical application of Qc suspension, Qc liposomes, and Qc optimum TFs, respectively. Drug pools were made by bottle caps with an area of $4.91 \mathrm{~cm}^{2}$ and this area was shaved carefully $24 \mathrm{~h}$ before application of the sample [24]. Half $\mathrm{ml}$ of each suspension was added to the drug pool. Scarification was done to rats at the end of the experiment. Overdose anesthetic ether was used to sacrifice three rats from each group at intervals of $(1,2,4,6,8,10 \mathrm{~h})$ and excision of the dorsal skin section that was in contact with the formulation was performed and washed three times with $10 \mathrm{ml}$ normal saline. Sonication with $5 \mathrm{ml}$ of methanol was performed to the excised skin sections after cutting into pieces for $30 \mathrm{~min}$. Filtration of the homogenized skin was done through a 0.45 $\mu \mathrm{m}$ filter membrane and an assay of Qc using HPLC was performed to determine its concentration. These data were used to calculate the skin deposition of Qc generated by the range of formulations tested. The animal carcasses were incinerated.

\section{Assay of Qc by HPLC}

A gradient HPLC method was chosen for the assay of Qc [25]. Waters 2690 Alliance HPLC system detector (America) was used, and the detector used was a UV detector. The type of column was $\mathrm{C}_{18}$ thermo column ( $4.6 \mathrm{~mm} \times 250 \mathrm{~mm})$. The temperature of the column was maintained at room temperature $\left(25.0 \pm 2.0^{\circ} \mathrm{C}\right)$. Mobile phase A was $0.1 \%$ Phosphoric acid and mobile phase B was acetonitrile. The flow rate was $0.5 \mathrm{ml} / \mathrm{min}$ and the UV detector was set at $262 \mathrm{~nm}$. Data analyses were made using SAS, (1999). Comparison of means was done using Duncan's at 5\% level of significance [26].

\section{Cutaneous wound induction and treatment}

Forty-eight male Wistar rats were used in the experiment. Anesthesia was made by intraperitoneal injection of ketamine and xylazine, and then shaving to the thoracodorsal part was done. A square-shaped 
wound $400 \mathrm{~mm}^{2}$ thick was created on the back of the rats using a scalpel blade and forceps [27]. After recovery from anesthesia animals were grouped in cages into 4 groups with twelve rats in each group. Treatment was done daily as follows: Group (I), Group (II), Group (III), and Group (IV) were treated with specified amounts of buffer (control), Qc suspension, Qc liposomes, and Qc optimum TFs, respectively. At the end of the experiment after $15 \mathrm{~d}$, the rats of each group were killed and the healing tissue was collected and divided into two portions. The first portion was stored at $-20^{\circ} \mathrm{C}$ and then homogenized in phosphate buffer saline ( $\mathrm{pH}=7.4)$. The tissue homogenates were centrifuged for $20 \mathrm{~min}$ at $12000 \mathrm{rpm}$ and $4{ }^{\circ} \mathrm{C}$ and the following biological parameters were estimated in the supernatants: GSH [28], MDA [29, 30], NO [31], TP [32], and TAC assay [33]. The second portion was used for the following histopathological examination.

\section{In vivo histopathological study}

The second portion from the above experiment was preserved in $10 \%$ formal saline and was used for the first histopathological study to compare the wound treatment effect of Qc suspension, Qc liposomes and Qc optimum TFs. Trimming of the samples was done and serial grades of ethanol were used for its processing, clearance in xylene, and then embedding them in paraffin beeswax blocks. Rotatory microtome was used to cut sections of $5 \mu \mathrm{m}$ thick. The sections were stained with Harris Hematoxylin and Eosin as a general examination method for histological evaluation. Masson's Trichrome stain was used for the demonstration of collagen fibers [34].

A second in vivo histopathological examination study using 16 male Wistar rats was performed to explore the irritation potential and the ultrastructural changes in the skin after topical application of the Qc optimum TFs and Qc liposomes formulation onto the skin three times a day for one week. Random division of rats was made into 4 groups; each group contained 4 animals: Group (I), group (II), group (III), and group (IV) for control, Qc suspension, Qc liposomes, and the Qc optimum TFs, respectively. At the end of the week, scarification of animals was done and excision of the skin was performed for histopathological examination according to the above protocol [34].

\section{RESULTS AND DISCUSSION}

\section{Characterization of Qc loaded TFs}

\section{Formulation variables effect on EE\%}

Entrapment of TFs of a significant amount of Qc is important for its use for topical application. Entrapment of Qc in TFs ranged from 85.80 to $98.38 \%$. Results of the entrapment efficiency in the TFsformulae are presented in table 2. 3-D plots in fig. 1 illustrate the effect of type of surface-active agent, lecithin to surface-active agent molar ratio, and hydration volume on EE\%. ANOVA results showed that only type of surface-active agent and hydration volume had a significant effect on $\mathrm{EE} \%(\mathrm{P}<0.0001)$.

This could be explained by the hydrophobicity of Qc. Hence, surface active-agent with low HLB values showed higher EE\% than surface active-agent with high HLB values. TFs containing Span 60 and Span 80 with HLB 4.7 and HLB 4.3, respectively, displayed EE\% higher than formulae containing Tween 60 and Tween 80 with HLB 14.9 and HLB 15, respectively. In addition, increasing hydration volume decreased the $\mathrm{EE} \%$. When the hydration volume increases, the drug entrapment in the lipid bilayers decreases, and its partition in the aqueous phase increases [35].

\section{Formulation variables effect on PS}

Small particles pass through skin more than larger ones [36, 37]. The results of PS of TFs are presented as Z average diameter in table 2 [38]. The PS of TFs composed of Tweens ranged in size between 674.2 and $1323.6 \mathrm{~nm}$. The influence of the type of surface-active agent, lecithin to surface-active agent molar ratio, and hydration volume is presented as 3D plots in fig. 1. Factorial analysis showed that only the type of surfaceactive agent had a significant effect on PS $(\mathrm{P}<0.0001)$.

Formulae from 9 to 16 containing Span 80 and Span 60 showed particle size higher than formulae from 1 to 8 containing Tween 60 and Tween80. This could be attributed to that Tweens have more deformability due to high flexibility and non-bulky hydrocarbon chains compared to Spans that have bulky hydrocarbon chains [39].

Table 2: Formulations, independent variables, and measured responses of the $2^{2} 4^{1}$ full factorial experimental design of TFs formulations

\begin{tabular}{|c|c|c|c|c|c|c|c|}
\hline \multirow[t]{2}{*}{ Formulations } & \multirow{2}{*}{$\begin{array}{l}X_{1} \\
\text { Type of surface- } \\
\text { active agent }\end{array}$} & \multirow{2}{*}{$\begin{array}{l}\mathrm{X}_{2} \\
\text { Lecithin to surface- } \\
\text { active agent molar ratio }\end{array}$} & \multirow{2}{*}{$\begin{array}{l}X_{3} \\
\text { Hydration } \\
\text { volume }(\mathrm{ml})\end{array}$} & \multirow{2}{*}{$\begin{array}{l}Y_{1} \\
\text { EE\%* }\end{array}$} & \multirow{2}{*}{$\begin{array}{l}Y_{2} \\
S^{*}(n m)\end{array}$} & \multirow{2}{*}{$\begin{array}{l}\mathrm{Y}_{3} \\
\text { PDI }^{*}\end{array}$} & \multirow{2}{*}{$\begin{array}{l}Y_{4} \\
P^{*}(m V)\end{array}$} \\
\hline & & & & & & & \\
\hline F1 & Tween60 & $5: 1$ & 10 & $90.40 \pm 0.56$ & $900.50 \pm 40.16$ & $0.65 \pm 0.16$ & $-8.32 \pm 1.47$ \\
\hline $\mathrm{F} 2$ & Tween 60 & $10: 1$ & 10 & $90.21 \pm 0.57$ & $1170.50 \pm 272.73$ & $1.33 \pm 0.22$ & $-9.24 \pm 0.26$ \\
\hline F3 & Tween60 & $5: 1$ & 20 & $88.70 \pm 0.98$ & $1323.60 \pm 679.30$ & $0.90 \pm 0.13$ & $-6.89 \pm 0.74$ \\
\hline $\mathrm{F} 4$ & Tween60 & $10: 1$ & 20 & $87.85 \pm 0.91$ & $795.85 \pm 51.68$ & $0.47 \pm 0.03$ & $-8.88 \pm 0.82$ \\
\hline F5 & Tween80 & $5: 1$ & 10 & $90.11 \pm 0.72$ & $960.00 \pm 345.06$ & $0.76 \pm 0.02$ & $-9.10 \pm 0.28$ \\
\hline F6 & Tween80 & $10: 1$ & 10 & $91.10 \pm 0.14$ & $695.35 \pm 20.29$ & $0.59 \pm 0.03$ & $-11.10 \pm 0.14$ \\
\hline F7 & Tween80 & $5: 1$ & 20 & $85.80 \pm 0.12$ & $1029.50 \pm 13.40$ & $0.72 \pm 0.24$ & $-8.22 \pm 0.40$ \\
\hline F8 & Tween80 & $10: 1$ & 20 & $86.60 \pm 0.56$ & $674.20 \pm 1.27$ & $0.51 \pm 0.00$ & $-10.43 \pm 1.23$ \\
\hline F9 & Span60 & $5: 1$ & 10 & $98.38 \pm 0.02$ & $3088.50 \pm 239.70$ & $0.67 \pm 0.45$ & $-15.45 \pm 1.76$ \\
\hline F10 & Span60 & $10: 1$ & 10 & $97.70 \pm 0.11$ & $2796.50 \pm 91.21$ & $1.00 \pm 0.37$ & $-15.70 \pm 0.70$ \\
\hline F11 & Span60 & $5: 1$ & 20 & $88.45 \pm 0.07$ & $2951.00 \pm 255.90$ & $0.73 \pm 0.00$ & $-18.45 \pm 1.34$ \\
\hline F12 & Span60 & $10: 1$ & 20 & $87.45 \pm 0.21$ & $2912.50 \pm 436.28$ & $1.00 \pm 0.31$ & $-18.15 \pm 1.48$ \\
\hline F13 & Span80 & $5: 1$ & 10 & $93.80 \pm 1.13$ & $2576.30 \pm 19.09$ & $0.77 \pm 0.47$ & $-18.61 \pm 1.83$ \\
\hline F14 & Span80 & $10: 1$ & 10 & $92.10 \pm 0.26$ & $3195.00 \pm 573.46$ & $0.66 \pm 0.47$ & $-18.90 \pm 0.98$ \\
\hline F15 & Span80 & $5: 1$ & 20 & $87.80 \pm 2.68$ & $3105.00 \pm 289.90$ & $0.72 \pm 0.38$ & $-17.55 \pm 0.49$ \\
\hline F16 & Span80 & $10: 1$ & 20 & $90.50 \pm 0.70$ & $3371.00 \pm 524.67$ & $0.75 \pm 0.34$ & $-16.95 \pm 0.49$ \\
\hline
\end{tabular}

${ }^{*}$ Data represented as mean $\pm \mathrm{SD}(\mathrm{n}=3)$. Abbreviations: EE\%, entrapment efficiency percent; PS, particle size; PDI, polydispersity index, and ZP, zetapotential.

\section{Formulation variables effect on PDI}

Wholly monodispersed particles have PDI of 0 , while highly polydispersed particles have PDI of 1 [40]. All TFs ranged from 0.335 to 1 . The influence of the type of surface-active agent, lecithin to surface-active agent molar ratio, and hydration volume is presented as 3-D surface plots in fig. 2. Factorial analysis showed tha tho factor had a significant effect on PDI (P>0.1).

\section{Formulation variables effect on ZP}

$\mathrm{ZP}$ is the measure of the total charges acquired by vesicles. Any formula is considered stable when the zeta potential value is around $\pm 30 \mathrm{mV}$ because of electrical repulsion between particles [41].
The obtained ZP values of TFs formulae ranged from-6.63 to-19.9 to $\mathrm{mV}$. The values of ZP of TFs formulae are presented in table 2. Because ZP was negative in all the formulations in our study, the discussion of variation in $\mathrm{ZP}$ will be in terms of its absolute value. The influence of the type of surface-active agent, lecithin to surface-active agent molar ratio, and hydration volume is presented as 3-D surface plots in fig. 2 . Factorial analysis showed that the type of surface-active agent and lecithin to surface-active agent molar ratio had a significant effect on zeta potential $(\mathrm{P}<0.0001$ and $\mathrm{P}<0.1$, respectively).

Decreasing lipophilicity of surface-active agents increased ZP due to the reduction of the surface free energy of the surface-active agent. Accordingly, formulae from 9 to 16 containing Span 60 and 
Span 80 with HLB 4.7and HLB 4.3, respectively, had higher ZP than formulae from 1 to 8 containing Tween 60 and Tween 80 with HLB 14.9 and HLB 15, respectively [42]. Also, the lecithin to surfaceactive agent molar ratio had a significant effect on ZP. The isoelectric point of lecithin is between 6-7; therefore, it is a zwitterionic compound since the $\mathrm{pH}$ of the saline buffer is 7.4, which is higher than the isoelectric point and since we use a nonionic surface-active agent, therefore, the net charge is negative therefore ZP is negative. The negativity of ZP was also due to the presence of hydroxyl ion on the surface of the vesicles [43]. When lecithin to surface-active agent molar ratio increased, ZP increased because the negative charge increased $[44,45]$.

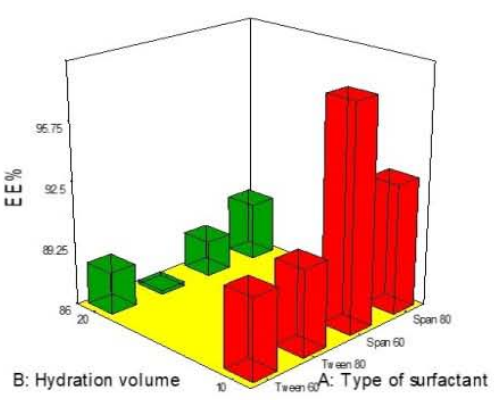

Actual Factor

C: Lecithin surfactant $=$ Average

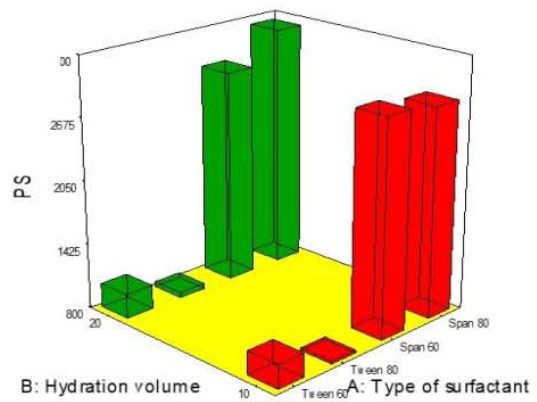

Actual Factor

C: Lecithin surfactant $=$ Averase
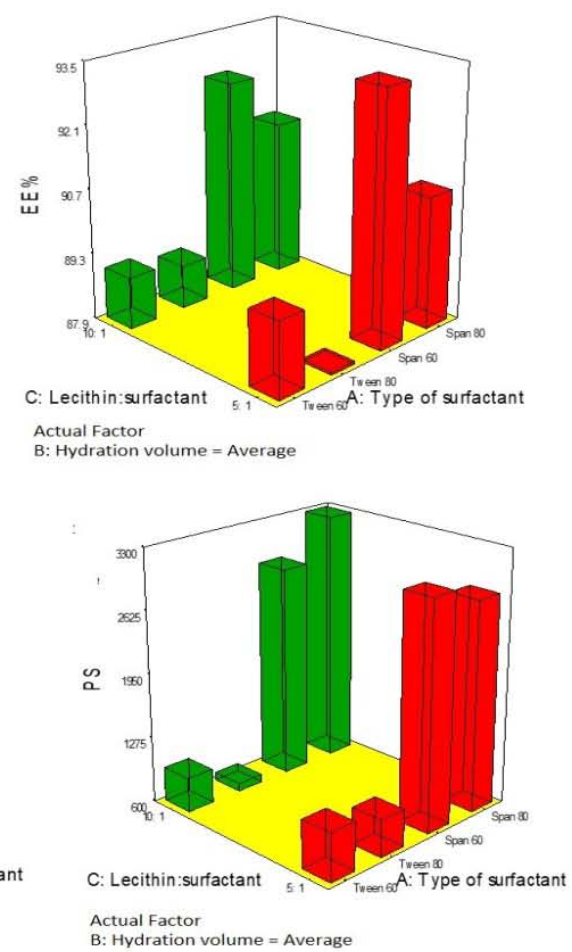

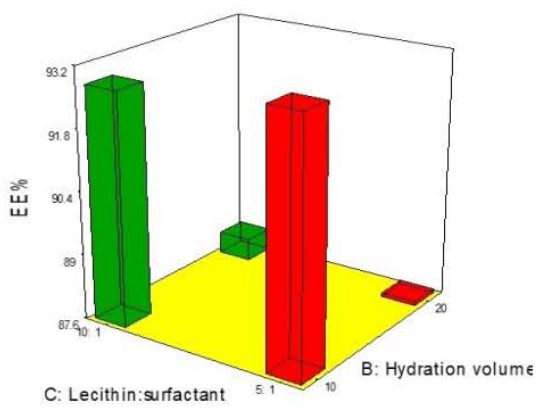

Actual Facto

A: Type of surfactant $=$ Average

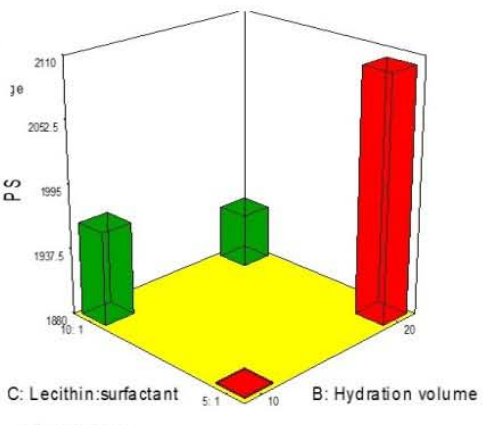

Actual Factor
A: Type of surfactant $=$ Average

Fig. 1: Response 3D-plots of the effect of type of surface-active agent, lecithin to surface-active agent molar ratio, and hydration volume on the EE\% and PS of TFs

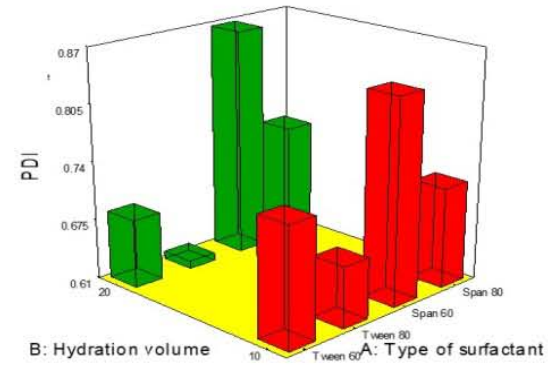

Actual Factor

C: Lecithin surfactant $=$ Average

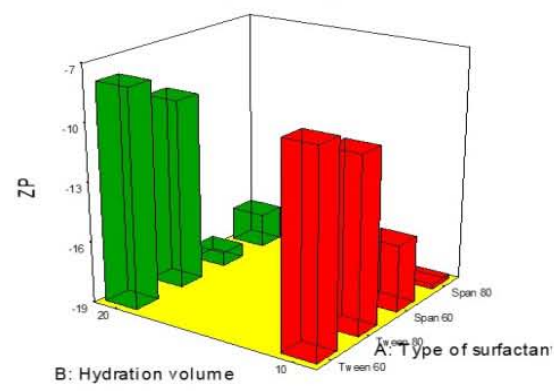

Actual Factor

$C$ : Lecithin surfactant $=$ Average

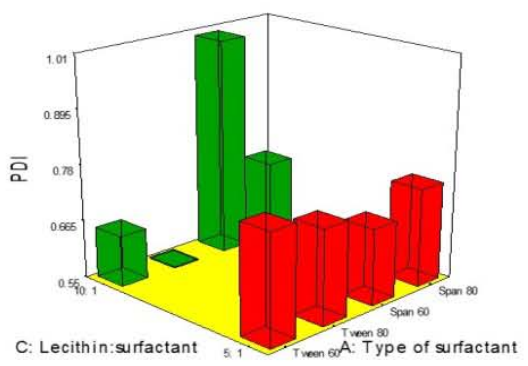

Actual Facto

B: Hydration volume $=$ Average

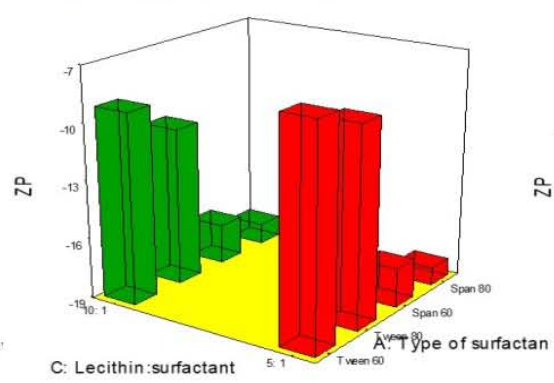

Actual Factor

B: Hydration volume $=$ Average

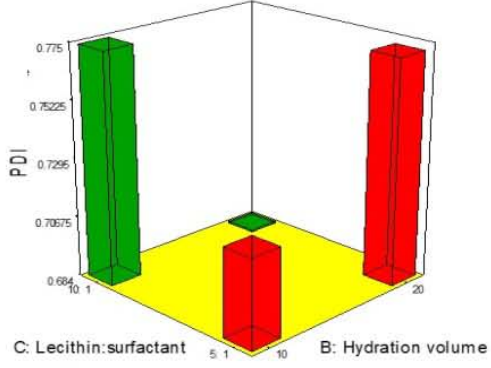

Actual Factor

A: Type of surfactant $=$ Average

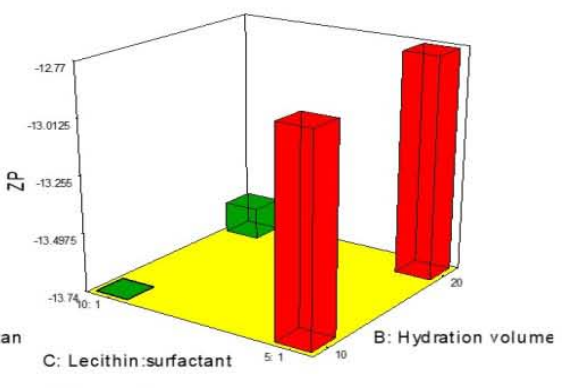

Actual Factor

A: Type of surfactant $=$ Average

Fig. 2: Response 3D-plots of the effect of type of surface-active agent, lecithin to surface-active agent molar ratio, and hydration volume on the PDI, and ZP of TFs 


\section{Optimum formulation}

Using Design Expert ${ }^{\circledR}$ Software, the optimum formulation was found to be F6. Choosing the optimum formulation was according to the following criteria (achieving the maximum value of $\mathrm{EE} \%$, minimum values of PS and PDI, and maximum value of $\mathrm{ZP}$ as absolute value). F6 was prepared with Tween 80, lecithin: surface-active agent molar ratio of $10: 1$, and hydration volume of $10 \mathrm{ml}$. This formulation showed EE\% of 91.1\%, PS of $695.35 \mathrm{~nm}$, PDI of 0.592, and ZP of- 11.1 $\mathrm{mV}$.

\section{TEM}

TEM analysis was used to verify the results of the Malvern analyzer of PS and also to examine the shape of the vesicular system [46]. Fig. 3 shows the TEM image of $\mathrm{F} 6$. The vesicles are non-aggregated, unilamellar, and spherical in shape. The size of the TEM agrees with that obtained from the Malvern particle size analyzer.

\section{DSC}

Fig. 4 shows the DSC thermogram of Qc, Tween 80, lecithin egg yolk, physical mixture of Qc with TFs components, and F6. Qc has two endothermic peaks at $122.5^{\circ} \mathrm{C}$ and $320^{\circ} \mathrm{C}$ [47]. Lecithin egg yolk has four endothermic peaks at $143.78{ }^{\circ} \mathrm{C}, 182.45{ }^{\circ} \mathrm{C}, 237.85{ }^{\circ} \mathrm{C}$, and $265.88{ }^{\circ} \mathrm{C}[48,49]$. Tween 80 shows one endothermic peak at 64.34 ${ }^{\circ} \mathrm{C}$ [50]. In the peaks of the physical mixture of Qc with the TFs constituents, the endothermic peak of Qc appears but less sharp due to its dilution with the excipients [51, 52]. In F6, the endothermic peak of Qc disappears due to inhibition of its re-crystallization and solubilization in TFs and its appearance in the more soluble and amorphous state, which leads to a high energy state which enhanced its solubility $[53,54]$.

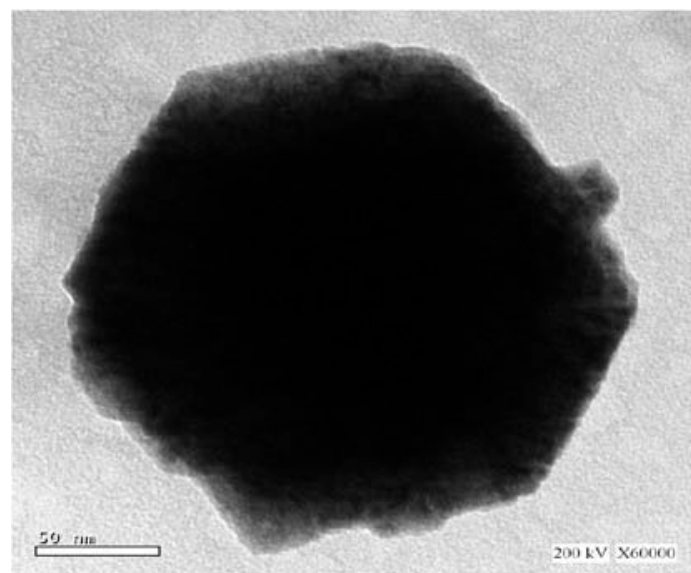

Fig. 3: TEM of Qc optimum TFs formulation (F6)

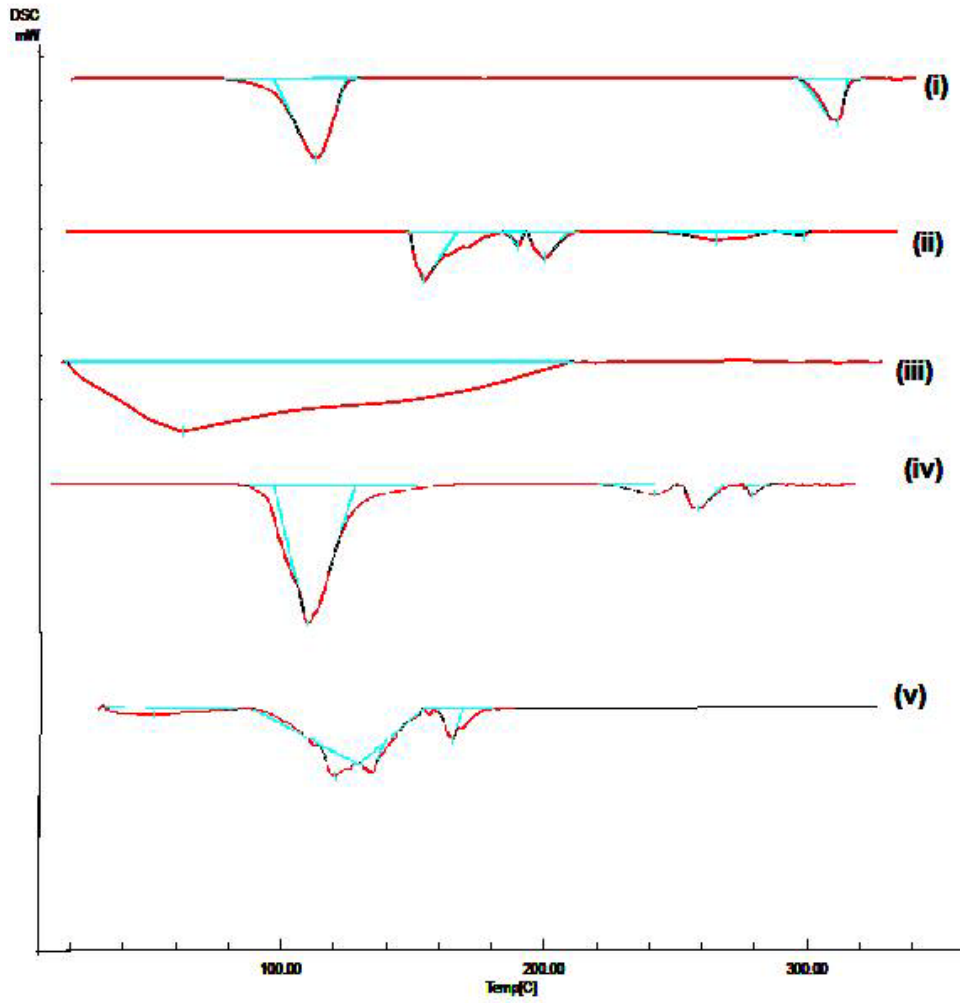

Fig. 4: DSC thermograms of (i) Qc, (ii) lecithin egg yolk, (iii) Tween 80, (iv) physical mixture, and (v) F6

\section{In vivo assessment of Qc loaded TFs}

\section{In vivo skin deposition experiment}

Fig. 5 shows the in vivo skin deposition profile from Qc suspension, Qc liposomes, and F6. Drug percentage retained in the skin from F6was significantly higher than Qc liposomes and Qc suspension at $2 \mathrm{~h}, 4 \mathrm{~h}, 6 \mathrm{~h}$, $8 \mathrm{~h}$, and $10 \mathrm{~h}(\mathrm{p}<0.05)$ (table 3). The percentage of Qc deposited from F6 in rat skin was 1.89 times higher than that from Qc liposomes and 4.3 times higher than Qc suspension. The higher Qc skin deposition from TFs could be due to the higher elasticity of TFs when compared to conventional liposomes as they have deformable properties, which can make them easily squeeze through the channels of the stratum corneum [55]. TFs are drug carrier systems that can penetrate the skin. The unimpeded moving of such carriers depends on two factors: the vesicle bilayers are very elastic and the presence of an osmotic gradient through the skin. Accordingly, TFs pass through the skin because their membrane is flexible, which prevents the rupture of the vesicles in the skin and permits the ultra-deformability of the TFs to change the composition of their membrane locally and reversibly when they are attracted to narrow pore. This decreases the percentage of membrane deformation and permits the flexible particles first to pass rapidly [56]. 


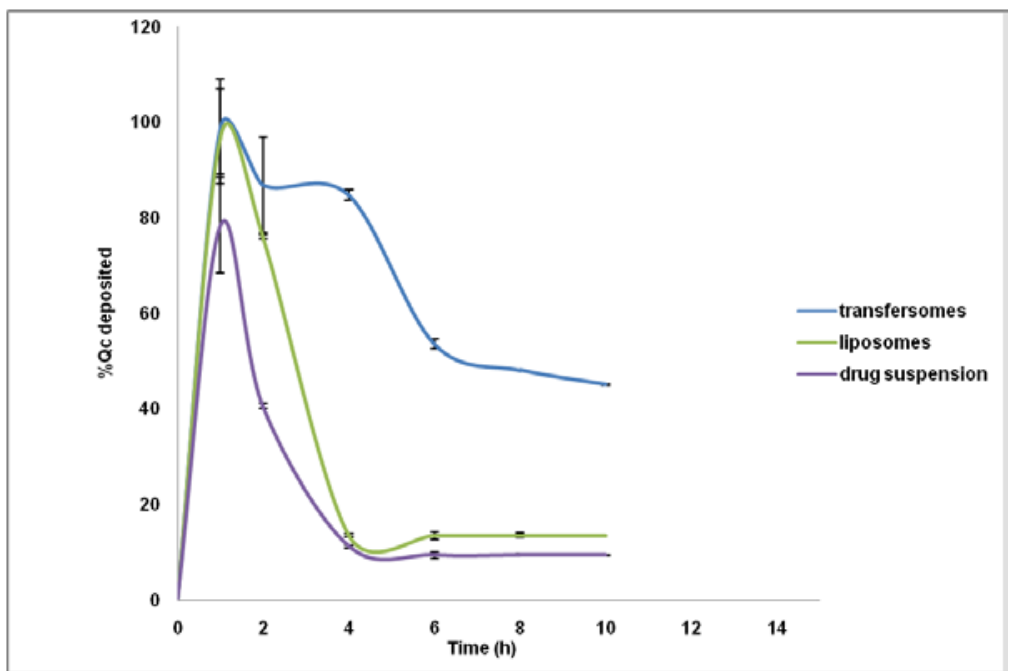

Fig. 5: In vivo deposition study of Qc suspension compared to Qc liposomes and Qc optimum TFs (F6)

Table 3: Statistical analysis of in vivo deposition study

\begin{tabular}{|c|c|c|c|c|c|c|}
\hline \multirow[t]{2}{*}{ \% Qc deposited } & \multicolumn{6}{|l|}{ Time (h) } \\
\hline & 1 & 2 & 4 & 6 & 8 & 10 \\
\hline Qc suspension & $78.50^{\mathrm{b}} \pm 10.00$ & $40.60^{\mathrm{d}} \pm 0.52$ & $11.20^{c} \pm 0.25$ & $9.40^{\mathrm{b}} \pm 0.72$ & $9.40^{c} \pm 0.23$ & $9.40^{c} \pm 0.06$ \\
\hline Qc liposomes & $97 \mathrm{a} \pm 10.00$ & $76^{c} \pm 0.40$ & $13.48^{\mathrm{c}} \pm 0.30$ & $13.48^{\mathrm{b}} \pm 0.90$ & $13.48^{\mathrm{c}} \pm 0.50$ & $13.48^{c} \pm 0.03$ \\
\hline F6 & $99^{a} \pm 10.00$ & $86.90^{\mathrm{a}} \pm 10.00$ & $84.74^{\mathrm{a}} \pm 1.10$ & $53.57 \mathrm{a} \pm 1.00$ & $48.10^{\mathrm{a}} \pm 0.10$ & $45.10^{a} \pm 0.10$ \\
\hline
\end{tabular}

Means in the same column with small letters are significantly different $(p<0.05), n=3 \pm S D$.

\section{Cutaneous wound induction and treatment}

\section{Biochemical parameters}

Wound induction undergoes an imbalance between production and accumulation of reactive oxygen species (ROS) in cells and tissues and this phenomenon is called oxidative stress and occurs by neutrophils which are derived from oxidants and MPO due to inflammation of wounds [57]. Biochemical parameters are shown in table 4.

Protein content in the wounded tissue represented the protein levels of cellular proliferation [58]. The difference between groups in the assay of TP was not significant $(p>0.05)$ [59]. TAC is an indication of wound healing. The difference in TAC between the control group and other groups was significant $(\mathrm{p}<0.05)$ as total antioxidant capacity increased in all treated groups. GSH is an antioxidant that captures free radicals; its increase is an indication for wound healing [60]. GSH decreased in the control group and increased in other groups. The difference between Qc liposomes and Qc suspension was not significant $(p>0.05)$. However, GSH in F6 group was significant compared to Qc liposomes and Qc suspension $(\mathrm{p}<0.05)$. NO is a free radical and its decrease indicates healing of wounds [61, 62]. NO increased in the control group and decreased in treated groups. The difference between Qc liposomes and Qc suspension was not significant $(p>0.05)$. However, NO in TFs was significantly lower than both of them $(\mathrm{p}<0.05)$. MDA is a natural marker of oxidative stress [63]. An increase in wound tensile strength increases MDA levels and mast cells [64]. After treatment of the wound, MDA and mast cells levels decrease as histamine increases [65]. MDA was high in the control group and low in the treated groups. There was no significant difference between the treated groups $(\mathrm{p}>0.05)$.

Table 4: Biochemical parameters in wound healed tissue following $15 \mathrm{~d}$ of topical application of Qc suspension, Qc liposomes, and F6

\begin{tabular}{llllll}
\hline Groups parameters & TP & TAC & GSH & NO & MDA \\
\hline Control & $2.80_{\mathrm{a}} \pm 0.18$ & $0.21_{\mathrm{b}} \pm 0.06$ & $9.51^{\mathrm{c}} \pm 0.64^{\mathrm{c}}$ & $60.00_{\mathrm{a}} \pm 7.07$ & $12.00_{\mathrm{a}} \pm 2.40$ \\
Qc suspension & $2.71_{\mathrm{a} \pm 0.55}$ & $0.27_{\mathrm{a}} \pm 0.07$ & $12.80_{\mathrm{b}} \pm 0.51$ & $38.10_{\mathrm{b}} \pm 1.56$ & $8.59_{\mathrm{b} \pm 0.97}$ \\
Qc liposomes & $2.69_{\mathrm{a}} \pm 0.16$ & $0.29_{\mathrm{a}} \pm 0.03$ & $12.61_{\mathrm{b}} \pm 0.41$ & $35.20_{\mathrm{b}} \pm 0.72$ & $8.41_{\mathrm{b} \pm 0.39}$ \\
F6 & $2.75_{\mathrm{a} \pm 0.48}$ & $0.30_{\mathrm{a}} \pm 0.07$ & $13.53_{\mathrm{a}} \pm 0.26$ & $21.00_{\mathrm{c} \pm 1.78}$ & $8.39_{\mathrm{b} \pm 0.17}$ \\
\hline
\end{tabular}

Mean in the same column with different small letters are significantly different $€ \rho .05), \quad(n=6 \pm S D)$ TP: Total protein, TAC: Total antioxidant capacity, GSH: Glutathione reduced, NO: Nitric oxide, and MDA: Malonaldehyde.

\section{Histopathological examination}

Fig. 6 shows the histopathological findings of the second section of the wound induction experiment. $\mathrm{H}$ and $\mathrm{E}$ stained wound sections were magnified at different levels. Control group (I) demonstrated retarded wound healing process with the persistence of a wide wound gap. Incomplete re-epithelialization due to immature epithelial layer and the presence of ulceration (arrow) with scab formed from necrotic tissue depresses and inflammatory cells infiltration (dashed arrow). Highly cellular granulation tissue with less mature collagen fibers is found in the dermis (star). Group treated with Qc suspension(II) demonstrated the same records as a control group with retarded wound healing process under scab (dashed arrow) with incomplete re-epithelialization and granulation tissue formation in dermal layer (star) accompanied with severe inflamed cells infiltrations and several congested and dilated deeper blood vessels (arrows). Group (III) treated with Qc liposomes showed incomplete healing with the persistence of a wide wound gap surrounded with granulation tissue (arrow) under scab from necrotic tissue depress and severe inflammatory cells infiltrates 
(star). Group (IV) treated with F6revealed the best wound healing with full re-epithelialization of the epidermis (arrow) and mature collagen made of fibers in the dermis with several hair follicles which were active and small amounts of inflamed cells. Remnant of old granulation tissue could be detected in the sub-epithelial region of the epidermis. Table 5shows the difference between groups in collagen percentage. Collagen percentage in F6 was significantly higher than control, Qc suspension, and Qc liposomes. Fig. 7 shows histopathological examination for collagen. F6 showed a higher inflammatory response, accelerated.

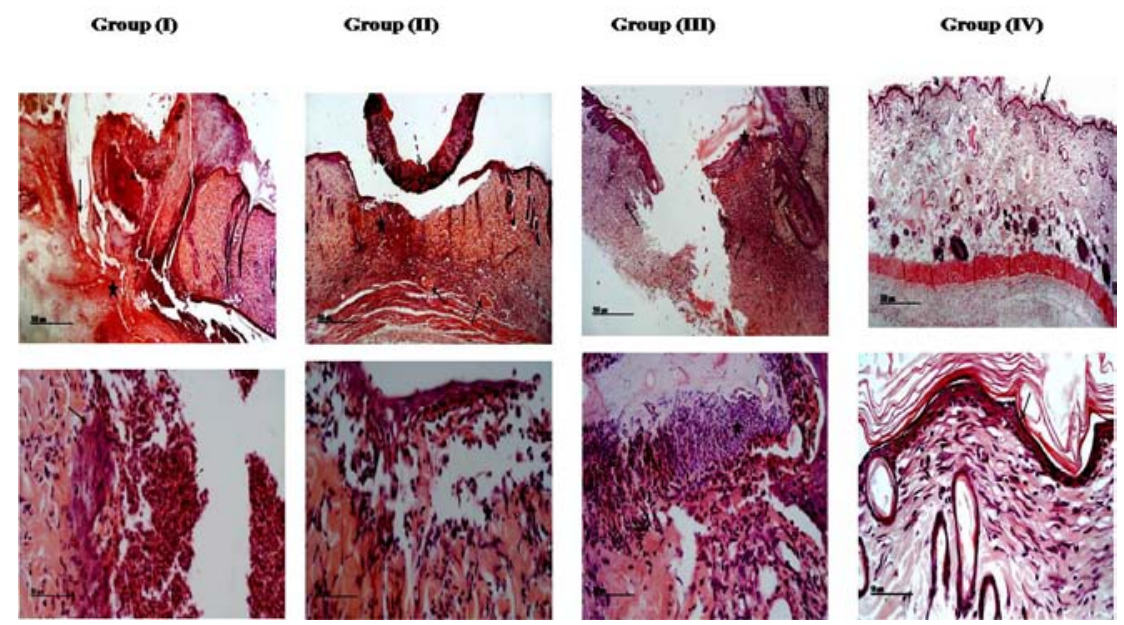

Fig. 6: Photomicrographs showing histopathological sections (Harris hematoxylin and eosin-stained) of treated groups after wound induction: the untreated control group (I), the group treated with Qc suspension (II), the group treated with Qc liposomes (III), and the group treated with F6 (IV)

Table 5: Collagen percentage in different groups after wound treatment

\begin{tabular}{lllll}
\hline $\begin{array}{l}\text { Area percentage of dermal } \\
\text { collagen fibers }\end{array}$ & Groups & & & \\
\cline { 2 - 5 } & Control & Qc suspension & Qc Liposomes & F6 \\
\cline { 2 - 5 } & $9.68^{\mathrm{d} \pm 0.79}$ & $10.60^{\mathrm{d}} \pm 1.20$ & $16.50^{\mathrm{c}} \pm 1.37$ & $35.20^{\mathrm{a}} \pm 1.44$ \\
\hline
\end{tabular}

Means in the same raw with different small letters are significantly different $(\mathrm{p}<0.05),(\mathrm{n}=6 \pm \mathrm{SD})$

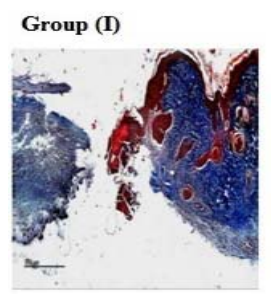

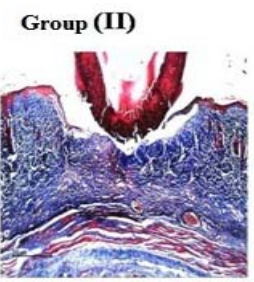
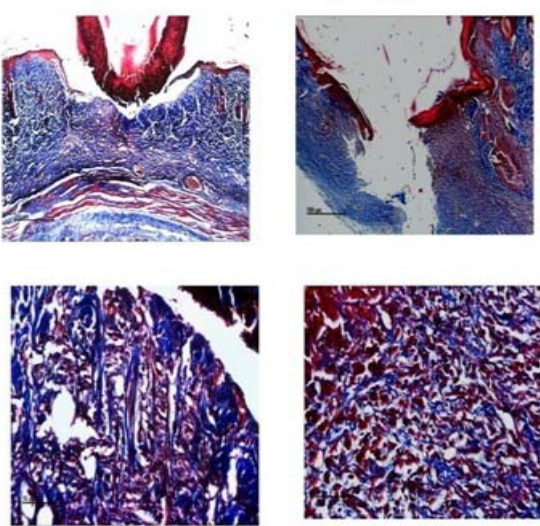

Group (III)

Group (IV)

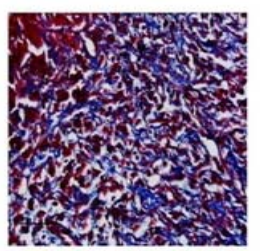

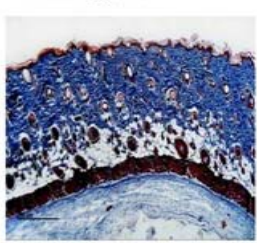

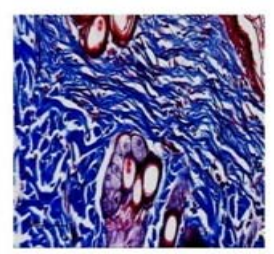

Fig. 7: Photomicrographs showing histopathological sections (Masson's Trichrome stain for demonstration of collagen fibers) of treated groups after wound induction: the untreated control group (I), the group treated with Qc suspension (II), the group treated with Qc liposomes (III), and the group treated with F6 (IV)

Wound healing and collagen percentage than Qc liposomes and Qc suspension as TFs were able to squeeze into the inside region of the outer layer of the skin (stratum corneum) and exhibit the anti-inflammatory and antioxidant activity because TFs are deformable so that it can go to the pores which are smaller than their size. On the contrary, Qc suspension and Qc liposomes didn't squeeze in the intercellular layer of the stratum corneum so they showed retardation in wound healing and antioxidant activity $[56,66]$.
For the second histopathological study, fig. 8shows microscopic pictures of rat skin sections that were stained: group (I), group (II), group (III), and group (IV) for control, Qc suspension, Qc liposomes, and F6, respectively. Microscopic examination of different skin samples from all groups revealed normal morphological features of different skin layers, including epidermis with intact keratinocytes, (Black arrows) dermis with mature collagen fibers, and many active hair follicles (red star) intact subcutaneous tissue muscles, and tissue (black star). No abnormal tissue alterations could be detected. 

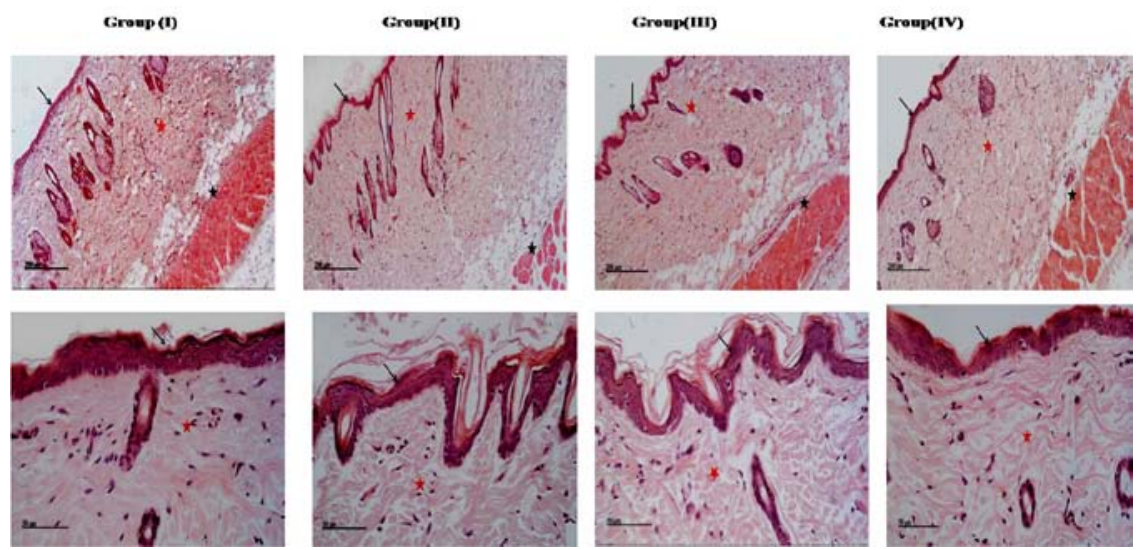

Fig. 8: Photomicrographs showing histopathological sections (Harris hematoxylin and eosin-stained) of the control group (I), the group treated with topical application of Qc suspension (II), the group treated with topical application of Qc liposomes suspension (III), and the group treated with topical application of F6 (IV)

\section{CONCLUSION}

Qc-loaded TFs were used topically for wound treatment. Statistical optimization of formulation variables was performed by factorial design using Design-Expert ${ }^{\circledR}$ software. The Qc optimum TFs (F6) formulation had a spherical shape, EE\% of $91.1 \%$, the particle size of $695.35 \mathrm{~nm}$, PDI of 0.592 , and $\mathrm{ZP}$ of $-11.1 \mathrm{mV}$. In vivo skin deposition experiment showed that F6was superior compared to Qc suspension and Qc liposomes. In addition, cutaneous wound treatment displayed that F6was superior to Qc suspension and Qc liposomes in wound treatment when assessed using biological parameters and histopathological studies. Furthermore, in vivo histopathological experiment confirmed that the topically applied F6 was not irritant. Therefore, the results proved that Qc-loaded TFs could be used as a successful drug delivery system to be used for wound healing.

\section{FUNDING}

Nil

\section{AUTHORS CONTRIBUTIONS}

All authors have contributed equally.

\section{CONFLICT OF INTERESTS}

\section{Declared none}

\section{REFERENCES}

1. Theng MA, GR Sitaphale, KR Biyani. Evaluation of wound healing activity of polyherbal formulation. Int J Curr Pharm Res 2017;9:12-4.

2. Awad MA, A de Jager, LM van Westing. Flavonoid and chlorogenic acid levels in apple fruit: characterization of variation. Sci Horticulturae 2000;83:249-63.

3. Pietta PG. Flavonoids as antioxidants. J Nat Prod 2000;63:1035-42.

4. Guardia T. Anti-inflammatory properties of plant flavonoids. Effects of rutin, quercetin and hesperidin on adjuvant arthritis in rat. Farmaco 2001;56:683-7.

5. Vijayalakshmi A, G Madhira. Anti-psoriatic activity of flavonoids from Cassia tora leaves using the rat ultraviolet $\mathrm{B}$ ray photodermatitis model. Rev Brasileira Farmacognosia 2014;24:322-9.

6. Sim GS. Structure-activity relationship of antioxidative property of flavonoids and inhibitory effect on matrix metalloproteinase activity in UVA-irradiated human dermal fibroblast. Arch Pharm Res 2007;30:290-8.

7. Bors W. Flavonoids as antioxidants: determination of radicalscavenging efficiencies, in methods in enzymology, Academic Press; 1990. p. 343-55.
8. Saija A. Flavonoids as antioxidant agents: the importance of their interaction with biomembranes. Free Radical Biol Med 1995;19:481-6.

9. Priyanga KS, VK. Investigation of antioxidant potential of quercetin and hesperidin: an in vitro approach. Asian J Pharm Clin Res 2017;10:83-6.

10. Sivapriya V. Novel nanocarriers for ethnopharmacological formulations. Int J Appl Pharm 2018;10:26-30.

11. Olivella MS. Effects of dimethyl and menthol, permeation enhancers: evaluation by skin microdialysis in rats. J Pharm Pharmacol 2007;50:49-54.

12. Tan $Q$, Preparation and evaluation of quercetin-loaded lecithinchitosan nanoparticles for topical delivery. Int J Nanomed 2011;6:1621-30.

13. Kazi KM. Niosome: a future of targeted drug delivery systems. J Adv Pharm Technol Res 2010;1:374-80.

14. Jain S, A Diwan, S Sardana. Use of lactic acid and span 80 in the formulation of lipid-based imiquimod vesicles for genital warts. Int J Pharm Pharm Sci 2017;9:292-301.

15. Gizaway SE. Betamethasone dipropionate gel for the treatment of localized plaque psoriasis. Int J Pharm Pharm Sci 2017;9:173-82.

16. Cevc G, A Schatzlein, H Richardsen. Ultradeformable lipid vesicles can penetrate the skin and other semi-permeable barriers unfragmented. Evidence from double-label CLSM experiments and direct size measurements. Biochim Biophysica Acta 2002;1564:21-30.

17. Elsayed MM. Lipid vesicles for skin delivery of drugs: reviewing three decades of research. Int J Pharm 2007;332:1-16.

18. Myers RH, DC Montgomery. Response surface methodology: product and process optimization using designed experiments. $2^{\text {nd }}$ Edition ed. John Wiley and Sons, New York; 2002.

19. Rogerson A. The distribution of doxorubicin in mice following administration in niosomes. J Pharm Pharmacol 1988;40:337-42.

20. Ichino T. Antitumor effect of liposome-entrapped adriamycin administered via the portal vein. Japan J Cancer Res 1990;81:1052-6.

21. Wang J. Nanoscale amphiphilic macromolecules as lipoprotein inhibitors: the role of charge and architecture. Int J Nanomed 2007;2:697-705.

22. Jain S. Proultraflexible lipid vesicles for effective transdermal delivery of levonorgestrel: development, characterization, and performance evaluation. AAPS PharmSciTech 2005;6:E513-22.

23. AOAC. Official method of Analysis. 20 $0^{\text {th }}$ Edition. Association of Officiating Analytical Chemists. Washington DC, Method; 2016. p. 935.14, 992.24.

24. Shen LN. Enhanced in vitro and in vivo skin deposition of apigenin delivered using ethosomes. Int J Pharm 2014;460:280-8. 
25. Begas E. Simple and reliable HPLC method for the monitoring of methotrexate in osteosarcoma patients. J Chromatogr Sci 2014;52:590-5.

26. Sendecor GW, WE. Cocharn statistical methods. $7^{\text {th. }}$ Press: Ames Aiwa, Aiwa Sate Univ; 1980. p. 507.

27. Primarizky H, Yuniarti WM, Lukiswanto BS. Ellagic acid activity in healing process of the incision wound on male albino rats (Rattus norvegicus). KnE Life Sci 2017;3:224-33.

28. Beutler E, O Duron, BM Kelly. Improved method for the determination of blood glutathione. J Lab Clin Med 1963;61:882-8.

29. Satoh K. Serum lipid peroxide in cerebrovascular disorders determined by a new colorimetric method. Clin Chim Acta 1978;90:37-43.

30. Ohkawa H, N Ohishi, K Yagi. Assay for lipid peroxides in animal tissues by thiobarbituric acid reaction. Anal Biochem 1979;95:351-8.

31. Montgomery HAC, Dymock JF. Colorimetric determination of nitrite. Analyst 1961;86:414

32. Gornall AG, CJ Bardawill, MM David. Determination of serum proteins by means of the biuret reaction. J Biol Chem 1949;177:751-66

33. Koracevic D. Method for the measurement of antioxidant activity in human fluids. J Clin Pathol 2001;54:356-61.

34. Bancroft OD, A Stevens. Theory and practice of histological technique. Chirchil Livingstone, Edinburgh. London and New york; 2010.

35. Batavia R. The measurement of beclomethasone dipropionate entrapment in liposomes: a comparison of a microscope and an HPLC method. Int J Pharm 2001;212:109-19.

36. Verma DD. Particle size of liposomes influences the dermal delivery of substances into skin. Int J Pharm 2003;258:141-51.

37. Plessis D. The influence of particle size of liposomes on the disposition of drug into the skin. Int J Pharm 1994;103:277-82.

38. Das S, WK Ng, RB Tan. Are nanostructured lipid carriers (NLCs) better than solid lipid nanoparticles (SLNs): development, characterizations and comparative evaluations of clotrimazoleloaded SLNs and NLCs? Eur J Pharm Sci 2012;47:139-51.

39. Cevc G. Transdermal drug delivery of insulin with ultradeformable carriers. Clin Pharmacokinet 2003;42:461-74.

40. Zeisig R, Shimada K, Hirota S, Arndt D. Effect of sterical stabilization on macrophage uptake in vitro and on thickness of the fixed aqueous layer of liposomes made from alkylphosphocholines. Biochim Biophys Acta 1285;1996:237-45.

41. Muller RH, C Jacobs, O Kayser. Nanosuspensions as particulate drug formulations in therapy. Rationale for development and what we can expect for the future. Adv Drug Delivery Rev 2001;47:3-19.

42. Abd-Elal RMA. Trans-nasal zolmitriptan novasomes: in vitro preparation, optimization and in vivo evaluation of brain targeting efficiency. Drug Delivery 2016;23:3374-86.

43. Abu El-Enin ASM. Proniosomal gel-mediated topical delivery of fluconazole: Development, in vitro characterization, and microbiological evaluation. J Adv Pharm Technol Res 2019;10:20-6.

44. Chain E, I Kemp. The isoelectric points of lecithin and sphingomyelin. Biochem J 1934;28:2052-5.

45. Pichot R, RL Watson, IT Norton. Phospholipids at the interface: current trends and challenges. Int J Mol Sci 2013;14:11767-94.
46. Lim WM. Formulation and delivery of itraconazole to the brain using a nanolipid carrier system. Int J Nanomed 2014;9:2117-26.

47. Anwer MK. Development and evaluation of PLGA polymer-based nanoparticles of quercetin. Int J Biol Macromol 2016;92:213-9.

48. Pannerselvam AKS AR. Isolation, characterization and formulation properties of new plant gum obtained from mangifera indica. Int J Pharm Biomed Res 2010;1:35.

49. SF Ibrahim ESE, Aa KES. Thermal analysis and characterization of some cellulosic fabrics dyed by a new natural dye and mordanted with different mordants. Int J Chem 2011;3:40.

50. Zafeiri I. The role of surface-active species in the fabrication and functionality of edible solid lipid particles. J Colloid Interface Sci 2017;500:228-40.

51. Avadhani KS. Skin delivery of epigallocatechin-3-gallate (EGCG) and hyaluronic acid loaded nano-transfersomes for antioxidant and anti-aging effects in UV radiation-induced skin damage. Drug Delivery 2017;24:61-74.

52. Sri KV. Preparation and characterization of quercetin and rutin cyclodextrin inclusion complexes. Drug Dev Ind Pharm 2007;33:245-53.

53. Basha MAEA S, Shamma R, Awad GJ. Design and optimization of surfactant-based nanovesicles for ocular delivery of clotrimazole. Liposome Res 2013;23:203-10.

54. Nasr AG A, Ghorab M. Novel solid self-nanoemulsifying drug delivery system (S-SNEDDS) for oral delivery of olmesartan medoxomil: design, formulation, pharmacokinetic and bioavailability evaluation. Pharmaceutics 2016;8:20.

55. Rajan R. Transferosomes-a vesicular transdermal delivery system for enhanced drug permeation. J Adv Pharm Technol Res 2011;2:138-43.

56. Rai S, V Pandey, G Rai. Transfersomes as versatile and flexible nano-vesicular carriers in skin cancer therapy: the state of the art. Nano Rev Exp 2017;8:1325708.

57. Song HS. The effect of caffeic acid on wound healing in skinincised mice. Korean J Physiol Pharmacol 2008;12:343-7.

58. Musalmah M. Comparative effects of palm vitamin E and alphatocopherol on healing and wound tissue antioxidant enzyme levels in diabetic rats. Lipids 2005;40:575-80.

59. Sinno $\mathrm{H}$. The effects of topical collagen treatment on wound breaking strength and scar cosmesis in rats. Canadian J Plastic Surgery 2012;20:181-5.

60. Weindl G. Hyaluronic acid in the treatment and prevention of skin diseases: molecular biological, pharmaceutical and clinical aspects. Skin Pharmacol Physiol 2004;17:207-13.

61. Boots AW. In vitro and ex vivo anti-inflammatory activity of quercetin in healthy volunteers. Nutrition 2008;24:703-10.

62. Schafer M, S Werner. Oxidative stress in normal and impaired wound repair. Pharmacol Res 2008;58:165-71.

63. Huxtable RJ. Physiological actions of taurine. Physiol Rev 1992;72:101-63.

64. Dincer S. Effect of taurine on wound healing. Amino Acids 1996;10:59-71.

65. Henderson WR, M Kaliner. Immunologic and nonimmunologic generation of superoxide from mast cells and basophils. J Clin Investigation 1978;61:187-96.

66. Fu J. Quercetin promotes diabetic wound healing via switching macrophages from M1 to M2 polarization. J Surgical Res 2020;246:213-23. 